\title{
Desenvolvimento de carreiras e perceção de fatores para a excelência do árbitro de futebol em Portugal \\ Desarrollo profesional y percepción de factores a la excelencia del árbitro de fútbol en Portugal Career development and perception of factors to the excellence of the football referee in Portugal Sérgio Mendes, Bruno Travassos, Ema Patrícia Oliveira \\ Universidade da Beira Interior (Portugal)
}

\begin{abstract}
Resumo. O processo formativo de um árbitro é longo, complexo e seletivo. Só um número reduzido de indivíduos atinge um patamar excelência que possibilita a direção de jogos nas grandes competições internacionais. Este estudo teve como objetivo identificar a carreira e o percurso dos árbitros portugueses de futebol que atingiram o nível de excelência e verificar as suas perceções sobre os fatores que estiveram na sua origem. Foram realizadas cinco entrevistas semiestruturadas junto de árbitros e ex-árbitros de futebol. Quatro deles atingiram a categoria de árbitro internacional de futebol e um deles, após ter abandonado a carreira de árbitro de futebol, atingiu a categoria de internacional no futsal. Foi adotado um design retrospetivo transversal. Após a recolha de dados procedeu-se a uma análise de conteúdo indutiva tendo emergido uma variedade de fatores pessoais e ambientais comuns a todos os participantes. Nos primeiros incluem-se os elementos de cariz: cognitivo, como o conhecimento do jogo, físicos, como a resistência ou a recuperação a fadiga, e psicológico, como a capacidade de regulação emocional. Nos fatores contextuais inserem-se, entre outros, a origem geográfica, a rede pessoal e institucional de apoio e o desenvolvimento de carreiras paralelas, como a académica ou a profissional. Os resultados sugerem que: 1) a carreira dos árbitros de futebol é marcada por quatro etapas distintas; 2) é possível identificar as exigências que definem a carreira de árbitros de futebol de excelência; 3) tendo por base as exigências identificadas, será possível melhorar o processo de formação e seleção dos árbitros de futebol.
\end{abstract}

Palavras-chave: árbitros de futebol, carreira, excelência, transições, dupla carreira.

Resumen. La excelencia en el arbitraje del fútbol es un objetivo buscado apenas por un número reducido de árbitros. Para que un árbitro alcance este pedestal se enfrenta a una variedad de exigencias, dentro y fuera del campo, y supera innumerables etapas en una carrera con dos o tres décadas aproximadamente. Este estudio ha tenido como objetivo identificar, por un lado, la carrera y el trayecto de los árbitros de fútbol portugueses que consiguieron el nivel de excelencia y por otro verificar sus percepciones sobre los factores que les permitieron alcanzar este pedestal. Se realizaron cinco entrevistas semiestructuradas, adoptando un diseño retrospectivo trasversal, a árbitros y ex árbitros de fútbol que consiguieron la categoría de internacional de fútbol o de fútbol sala. Después de recoger los datos se procedió a un análisis inductivo y así emergieron una variedad de factores personales y ambientales comunes a todos los participantes. En los primeros se incluyen los elementos de carácter cognitivo, como el conocimiento del juego, físico y psicológico, como la capacidad de regulación emocional. En los factores contextuales se incluyen, entre otros, el origen geográfico, la red personal e institucional de apoyo y el desarrollo de carreras paralelas, como la académica o la profesional. Los resultados de la investigación: 1) Sugieren el dinamismo de la carrera de los árbitros de fútbol, a través de la influencia de los factores referidos, marcados por la exigencia de cuatro etapas distintas; 2) Permite una mejor caracterización de las exigencias que definen la carrera de un árbitro de fútbol; 3) Permiten ayudar a ajustar a las entidades que gestionan el arbitraje nacional el proceso de formación y selección de los árbitros de fútbol.

Palabras-clave: árbitros de fútbol, carrera, excelencia, transiciones, doble carrera.

\begin{abstract}
The excellence in soccer refereeing is a goal attained only by a small number of referees. For a referee to reach this level, he challenges several demands, both in and out of the field, and overcomes some stages in a career spanning approximately two to three decades. This study aimed to identify the career and the path/development of the Portuguese soccer referees who reached the level of excellence and assess their perceptions about the determinant/decisive factors to reach that level. Five semi-structured interviews were conducted with referees and former soccer referees who reached the international category, in football or futsal, using a transverse retrospective design. After finishing the data collection, an inductive analysis was carried out and a several personal and environmental common factors were identified. A list of these include: cognitive elements as the game's knowledge/laws of the game knowledge, physical elements, as endurance, recovery and tiredness, and psychological elements, as the ability/skill of emotional regulation. In the context factors are included, among others, region/place of origin, personal and institutional network of support and the development of parallel careers, such as academic or professional. The results suggest/state: 1 ) the presence of four different stages in a football referee career; 2) allow a better characterization of the requirements that define excellence in the context of a football referee career; 3) allow national entities, responsible for the selection and training of football referees, to improve their processes/methods.
\end{abstract}

Keywords: career, dual career, excellence, football referees, transitions

\section{Introdução}

Como fenómeno à escala mundial o futebol tem, simultaneamente, um grande impacto na dimensão emocional dos seus adeptos e na aposta financeira dos seus investidores (Catteeuw, Gilis, Wagemans, \& Helsen, 2010). O árbitro, pela especificidade da sua função e pelo papel de

Fecha recepción: 15-02-19. Fecha de aceptación: 26-09-19

Sérgio Miguel Cardoso Mendes

sergioalbicastrense@gmail.com mediação e de decisão que assume no contexto do jogo, assume uma posição paradoxal. Embora se pretenda o mais discreto possível é frequentemente a personagem principal do jogo por causa da importância das suas decisões e das consequências das mesmas (Slack, Maynard, Butt, \& Olusoga, 2013).

A comunidade científica tem estado atenta a esta realidade. Só na última época assistiu-se a um boom de publicações sobre o tema da arbitragem. No entanto, a maioria está focada apenas na caraterização do desempenho físico e 
técnico, negligenciando todos os outros aspetos que caraterizam a prestação e desenvolvimento de capacidades do árbitro de futebol (Aragão e Pina, Passos, Araújo, \& Maynard, 2018). Ainda assim, são vários os estudos que reforçam que para responder às exigências do jogo o árbitro necessita desenvolver um conjunto diversificado de capacidades e competências (Krustrup et al., 2009; Slack et al., 2013), que não se cingem ao domínio das leis de jogo (Constantin, 2015) ou uma boa condição física (Castillo, Javier, Casajús, \& Cámara, 2016). O árbitro deverá ter a capacidade de tomar decisões em tempos reduzidos (Catteeuw, Helsen, Gilis, \& Wagemans, 2009) que não sejam enviesadas por fatores externos (Dohmen \& Sauermann, 2016) tais como, pela pressão social (Dohmen, 2008), pelo comportamento dos jogadores (Folkesson, Nyberg, Archer, \& Norlander, 2002) ou mesmo dos adeptos (Boyko, Boyko, \& Boyko, 2007). O árbitro deve ainda dominar estratégias de comunicação (Furley \& Schweizer, 2016) uma vez que a forma como se expressa influi na consistência como é percebida a confiança nas suas decisões.

No percurso para o desenvolvimento destas capacidades e competências, à semelhança de um desportista de elite, um árbitro tem de superar um conjunto de etapas e de eventos de mudança (Samuel, Galily, \& Tenenbaum, 2015; Stambulova, Alfermann, Statler, \& Côté, 2009), num processo que depende em muito de si (Philippe, Vallerand, Andrianarisoa, \& Brunel, 2009; Slack et al., 2013), mas cuja influência contextual não pode ser ignorada (Kizilet, 2011; Racek \& Pelikán, 2015; Webb, Wagstaff, Rayner, \& Thelwell, 2016). É nesta perspetiva que o apoio institucional para o desenvolvimento da carreira de árbitro tem também despertado algum interesse nos últimos anos (Sarmento, Marques, \& Pereira, 2015). Por exemplo, o processo formativo (Gallardo \& Iglesias, 2011; González Oya, 2005, 2006), a retenção / recrutamento de novos árbitros (Perreau-niel \& Erard, 2015; Wicker \& Frick, 2016) ou a análise acerca da estrutura institucional de apoio ao desenvolvimento do processo formativo e seletivo dos árbitros de futebol (Webb, 2017; Webb et al., 2016) foram temáticas abordadas.

Tendo por base os modelos de desenvolvimento e transição de carreiras desportivas, (Samuel et al., 2015; Stambulova et al., 2009), no que diz respeito ao estudo do percurso dos árbitros até ao nível de excelência, Slack, et al (2013) procuraram identificar os fatores percebidos para sustentar a excelência na arbitragem de futebol. Os resultados sugerem a existência de uma variedade de fatores físicos, ambientais e psicológicos, de entre os quais, (a) atributos de resistência mental; (b) redes e serviços de apoio; (c) qualidades de gestão de jogo; (d) preparação pré-jogo multifacetada, (e) busca pela melhoria do nível de desempenho, (f) oportunidades de prosperar, (g) características pessoais e (h) componentes físicos superiores, que caraterizam claramente os árbitros de excelência. Noutro sentido a investigação de Samuel et al (2015) procurou definir a carreira profissional do árbitro de futebol, sugerindo a existência de uma carreira dinâmica que compreende uma série de eventos de mudança relacionados com diferentes aspetos como: (a) o desempenho no exercício de funções; (b) o desenvolvimento de carreira; (c) o apoio institucional; (d) questões de vida em geral. No que concerne ao seu desenvolvimento, a carreira de árbitro é iniciada nos escalões regionais de formação, sendo que apenas os mais talentosos e qualificados continuam uma trajetória como árbitros principais e atingem os patamares mais elevados. Os restantes permanecem em escalões inferiores ou, perante a incapacidade de progredir, mudam para o percurso para o de árbitros assistentes.

Apesar da existência de alguns estudos que procuraram traçar o desenvolvimento da carreira dos árbitros (Weston et al., 2011) e identificar os fatores que sustentam a excelência na arbitragem, escasseiam trabalhos que explorem o percurso dos árbitros numa perspetiva holística e não meramente desportiva (Samuel et al., 2015). Os resultados das investigações conhecidas sobre atletas apontam para a importância de aspetos de natureza contextual como a origem geográfica (Côté, Macdonald, Baker, \& Abernethy, 2006), ou da influência familiar (Côté, 1999; Côté \& Vierimaa, 2014). No domínio da arbitragem é sugerida a importância do suporte familiar (Perreau-niel \& Erard, 2015) e do apoio institucional (Kizilet, 2011; Sarmento et al., 2015; Webb, 2017), mas ainda sem quaisquer evidencias. Do mesmo modo, tal como se verifica na prática desportiva de alta competição, alguns estudos apontam para a existência de vantagens no desenvolvimento de carreiras duais como fator diferenciador no desenvolvimento desportivo e individual dos indivíduos (Carless \& Douglas, 2013). No entanto, na arbitragem em geral e em particular na arbitragem no futebol, nada se sabe.

Face ao exposto, este estudo teve como objetivos: i) analisar as etapas da carreira dos árbitros portugueses de futebol que atingiram o nível de excelência (árbitro internacional) e os seus diferentes percursos; ii) verificar a perceção dos participantes sobre os fatores que permitiram alcançar o patamar de excelência.

\section{Metodologia}

\section{Participantes}

Participaram voluntariamente neste estudo cinco árbitros / ex-árbitros Portugueses de futebol $(M=49.6, D P=6.87$ idade; $M=16.6, D P=2.30$ anos de experiência como árbitro $1^{\text {a }}$ categoria; $M=12.2, D P=2.04$ ). Quatro deles atingiram a categoria de árbitro internacional de futebol e um deles, após ter abandonado a carreira de árbitro de futebol, atingiu a categoria de internacional no futsal. No momento da entrevista, apenas um dos árbitros ainda se encontrava no ativo, dois tinham-se retirado há mais de cinco anos e dois há menos de cinco anos. Como atividade profissional, um dos participantes desempenhava a função de árbitro profissional, um era comentador desportivo, e dois estavam ligados à estrutura dirigente / formativa da arbitragem, havendo ainda um que não mantinha qualquer relação com a arbitragem. Em termos do seu contexto de origem, os entrevistados eram originários de associações de futebol de grande $(n=2)$, média $(n=2)$ e pequena dimensão $(n=1)$, sendo que quatro eram originários de associações de futebol do litoral e um do interior do país.

\section{Instrumento}

Foi adotado um guião de entrevista semiestruturado, procurando abranger as temáticas centrais inerentes à 
excelência na arbitragem de futebol e, ao mesmo tempo, capturar as experiências individuais de cada entrevistado em relação ao fenómeno em estudo. De forma a ir ao encontro dos objetivos previamente definidos, o guião de entrevista foi organizado em torno de dois grandes temas - (a) características pessoais e (b) percursos (escolar, profissional, desportivo e como árbitro) de modo a traçar as diferentes carreiras dos entrevistados, identificar as caraterísticas pessoais e explorar os aspetos contextuais que sustentaram a sua carreira. O guião resultou de uma adaptação de outros instrumentos utilizados em investigações anteriores que remetem para abordagens compreensivas da excelência (Araújo, Cruz, \& Almeida, 2011; Côté, Ericsson, \& Law, 2005; Dias, Cruz, \& Fonseca, 2009; Holt \& Dunn, 2004).

\section{Recolha de dados}

As abordagens qualitativas têm sido valorizadas enquanto opção metodológica na investigação sobre a excelência (Holt \& Dunn, 2004; Jalil \& Boujettif, 2005). De acordo com Simonton (1999), «individuals who are the best exemplars of a particular form of creativity, leadership, or other talent are most likely to represent statistical outliers with respect to relevant variables» (p. 440). Nesta linha, seguindo uma lógica ideográfica, procura-se mais a compreensão aprofundada dos fenómenos do que a sua generalização. Entre as formas diretas de recolha de dados, a entrevista adquire particular relevância quando se trata de compreender as idiossincrasias dos indivíduos excecionais, apesar da dificuldade muitas vezes encontrada em conciliar agendas com vidas profissionais de sucesso bastante preenchidas. Assim, a interpretação dos dados recolhidos deverá ter em consideração as características dos participantes e, em última análise, o investigador poderá não ter qualquer interesse que os dados obtidos com indivíduos de excelência sejam significativos e generalizáveis para a generalidade da população (Simonton, 1999).

Atendendo às considerações anteriores, todas as entrevistas foram iniciadas com uma explicação sucinta dos objetivos da investigação, a garantia de confidencialidade dos dados, procurando-se desta forma, garantir o estabelecimento de uma relação de confiança entre o participante e o investigador

As entrevistas duraram, em média, 49.40 \pm 16.25 min e a sua transcrição literal pelo autor deu origem a 52 páginas de espaço simples. Foram aplicados pseudónimos para garantir o anonimato de todas as informações de identificação pessoal. Após a transcrição literal as entrevistas foram devolvidas por mail aos participantes para que confirmassem a qualidade e a precisão do seu conteúdo e significado. Apenas um participante prestou informações adicionais que não provocaram uma alteração significativa na transcrição realizada.

\section{Análise dos dados}

Nesta etapa foi definido um sistema de codificação preliminar assente na abordagem dedutiva e, por conseguinte, na literatura existente, sendo definidas as categorias (Robbins \& Judge, 2013), assim como algumas subcategorias (Oh, Wang, \& Mount, 2011). O primeiro sistema foi organizado em três níveis hierárquicos: (a) dimensões gerais; (b) dimensões específicas; (c) categorias. Foram consideradas como dimensões gerais: 1) percursos; 2) Influência dos diversos elementos na carreira como árbitro. Antes de se iniciar o processo de codificação foi definido o respetivo protocolo. Foi entendido analisar uma entrevista de cada vez (Schilling, 2006), incidindo a análise sobre o conteúdo manifesto, que remete para a interpretação literal, assim como no conteúdo latente, ou seja, na interpretação do significado subjacente (Bardin, 2004; Graneheim \& Lundman, 2004). Relativamente à categorização ficou estabelecido adotar os procedimentos definidos por Richards (2009) e Krippendorff (1980).

Durante o processo de codificação realizaram-se diversas reuniões entre os pesquisadores para proceder à análise e comparação das codificações. Tal facto deu origem a algumas alterações à proposta inicial, principalmente aquando da definição das subcategorias, seguindo-se desta forma um processo indutivo de análise. Estas mudanças resultaram do cruzamento de informações entre os diferentes codificadores, um procedimento que procurou assegurar maior rigor na análise de conteúdo efetuada. Por fim, foram calculados os valores percentuais das dimensões específicas a partir do total de codificações da dimensão geral. A percentagem de cada uma das categorias baseou-se no total de codificações da dimensão específica, enquanto o das subcategorias resultou da soma total de codificações da categoria que integram.

\section{Resultados}

A análise de conteúdo das cinco entrevistas deu origem a um total de 408 codificações, organizadas em duas dimensões gerais: Percursos e Influência dos diversos elementos na carreira como árbitro.

\section{Percursos}

Os resultados da dimensão geral «Percursos» apontam para a existência de cinco dimensões específicas que caraterizam os percursos dos árbitros de futebol (ver Figura 1). Por ordem de representatividade de unidades de significado: (i) a dimensão «Desportivo» que apresenta as categorias «prática desportiva antecedente», «Iniciação / integração na arbitragem», «Desenvolvimento», «Manutenção» «Fim da carreira de árbitro»; (ii) a dimensão «Profissional» que apresenta como categorias «Inicial», «desenvolvida durante a carreira de árbitro» e «Após retira-

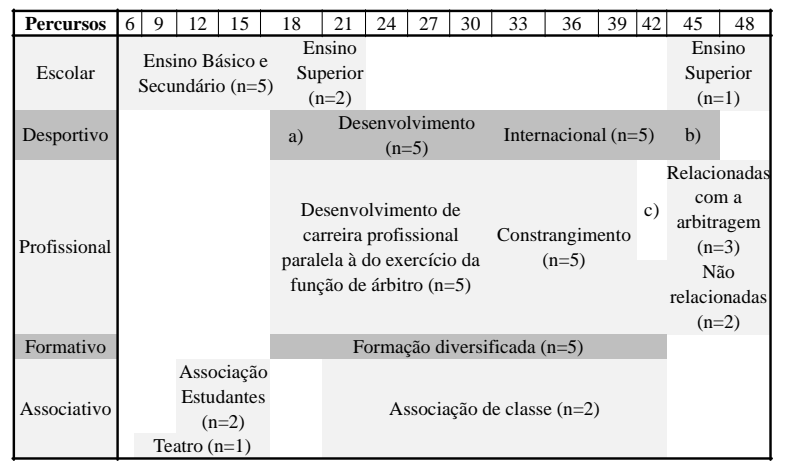

Nota - a) Integração (n=5); b) Retirada como árbitro (n=4); c) Fim do percurso profissional não relacionado com o desporto $(\mathrm{n}=3)$

Figura 1 - Cruzamento por ordem cronológica dos percursos dos entrevistados. 
da da carreira de árbitro»; (iii) a dimensão «Escolar» que apresenta a categoria «nível de escolaridade»; (iv) a dimensão «Formativa» que apresenta as categorias «relacionadas com o desporto» e «não relacionadas diretamente com o desporto»; (v) a dimensão específica «associativo» que apresenta as categorias «arbitragem» e «não relacionadas com a arbitragem».

De modo a responder ao primeiro objetivo - analisar as etapas de desenvolvimento da carreira dos árbitros de futebol portugueses que atingiram o nível de excelência (árbitro internacional) e os seus diferentes percursos - foram avaliadas as unidades de significado de cada dimensão específica, tendo resultado na Tabela 1. Os resultados têm por base o cruzamento das idades indicadas pelos participantes nas diferentes etapas das suas vidas com os seus percursos escolar, profissional, formativo e associativo.

Os resultados revelaram que todos os participantes realizaram um percurso escolar regular até ao ensino secundário, sendo que apenas dois prosseguiram estudos no ensino superior e apenas um os retomou após a sua retirada como árbitro. Desportivamente a carreira foi iniciada por todos a partir dos 10 anos em diversas modalidades. $\mathrm{O}$ percurso na arbitragem iniciou-se, de forma fortuita, na transição para a segunda década de vida. Após este momento verificou-se uma ascensão progressiva de cerca de 12 anos até ao patamar de árbitro internacional. Os participantes mantiveram-se neste patamar até à sua retirada, cerca dos 45 anos. Do ponto de vista profissional o percurso iniciouse aos dezoito anos. A partir dos 36 anos todos os participantes relataram a existência de constrangimentos profissionais que, aos 42 anos, obrigou dois a colocar fim ao seu percurso profissional em atividades não relacionadas com o desporto. Dois entrevistados referiram ter iniciado, após o fim da carreira como árbitro, percursos profissionais

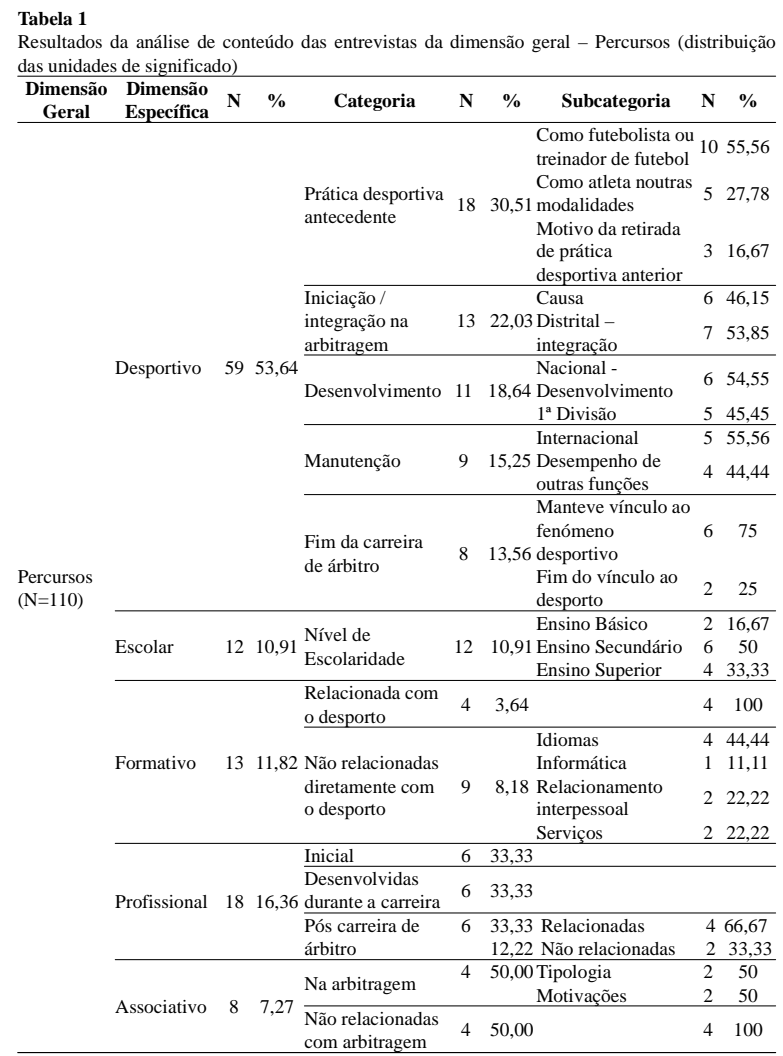

distintos da atividade desportiva anterior, enquanto três indicaram que se encontram a desenvolver atividades relacionadas com a arbitragem e o futebol. O percurso formativo de todos os participantes decorreu em paralelo com a carreira profissional e a de árbitro, estando diretamente relacionado com o exercício destas funções. Por fim, no percurso associativo, verificou-se que o início da atividade de árbitro, por volta dos 20 anos, marca o fim do envolvimento em atividades associativas não relacionadas com a arbitragem de dois participantes. Constata-se que, pouco tempo depois, pelos 23 anos outros dois árbitros indicaram ter integrado projetos relacionados com a sua associação de classe, principalmente depois de atingirem o patamar nacional mais elevado. Paralelamente referiram que mantiveram esta condição até à sua retirada.

\section{Influência dos diversos elementos na carreira como árbitro}

Relativamente ao segundo objetivo, verificámos as perceções dos participantes sobre os fatores pessoais e contextuais que influíram no desenvolvimento do percurso dos árbitros de futebol. Neste sentido privilegiámos o número de unidade de significados codificado e apresentados na Tabela 2 por revelar o peso atribuído pelos participantes a determinados fatores. Os resultados revelaram uma perceção menos significativa aos aspetos de cariz interno em comparação com os aspetos de cariz externo. Em relação aos aspetos de cariz interno, a categoria «desempenho», principalmente no desenvolvimento do processo revelou-se como sendo uma das consideradas de maior importância no desenvolvimento da carreira.

No meu tempo eu procurava ter uma vida regrada, uma preparação cuidada, treinar diariamente, com treino de recuperação à segunda, normal à terça, de descanso à quarta,

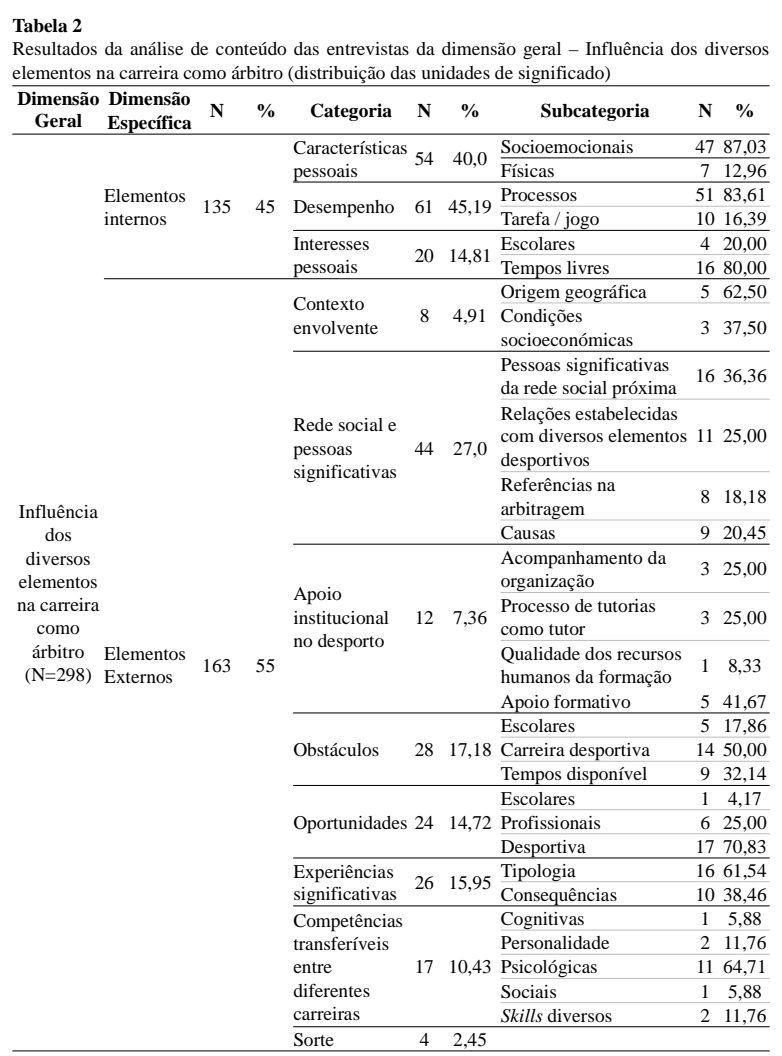


treino técnico na quinta e, consoante o dia do jogo, descansava ou viajava. Pelo meio tinha uma parte técnica, de estudo, nos núcleos

Árbitro 3

Quando me perguntam o que foi importante para a carreira: o treino, a relação com o treino e a relação com o núcleo foram também determinantes.

Árbitro 4

As caraterísticas pessoais, em especial as de cariz socioemocional foram também fortemente referidas pelos participantes. De entre estas foi realçado, entre outras, o perfecionismo, a exigência, o rigor ou a resiliência.

...considerei-me rigoroso e exigente, principalmente comigo mesmo...

Árbitro 2

...uma grande capacidade de sacrifício e sofrimento, e saber resistir às agressões que vêm do meio.

Árbitro 3

Os interesses pessoais surgem num patamar inferior, sendo referidos os tempos livres, e sobretudo os relacionados com atividades de futebol ou arbitragem também considerados importantes no desenvolvimento das suas carreiras.

Por sua vez, nos aspetos de cariz externo, emerge como categoria mais significativa «Rede social e pessoas significativas». Nesta categoria, a referência a relações significativas na rede social ou relações estabelecidas assumem elevada preponderância.

A estabilidade familiar é importante, tal como a estabilidade emocional. (...) Tenho a sorte de ter uma família que coloca a arbitragem em primeiro lugar.

Árbitro 5

Existem ainda 3 categorias com nível de significância idêntico: «obstáculos» de onde sobressaem os obstáculos «desportivos» e capacidade para os contornar; «experiências significativas» de onde emerge a «tipologia» dessas experiências; e por fim, a categoria «oportunidades» de onde se destacam as «desportivas».

Na época dizia-se e sentia-se que o ambiente em torno da arbitragem não era muito credível. Essa era a verdade que se veio a provar anos mais tarde. Havia muitos lobbys, muitos crimes não provados de influências, de trocas de favores.

Árbitro 2

$\mathrm{O}$ pior jogo que eu tive foi marcado por erros resultantes do cansaço do sistema nervoso central. (...) Estava cansado devido aos muitos jogos. Ainda não tinha recuperado do cansaço do mundial. (...) Nessa semana treinei de segunda a quinta-feira sem descanso (...) Cheguei mesmo a solicitar apoio psicológico e a realizar exames oftalmológicos.

Árbitro 4

Integrei um programa-piloto. Fui terceiro classificado a nível nacional nesse curso e tive o prazer de ter participado no Torneio Internacional do Porto.

Árbitro 3

Com um nível de significância menor surge a categoria «Competências transferíveis entre as diferentes carreiras», sendo de destacar as competências de cariz psicológico. Por último, surgiu a categoria o "Apoio institucional no desporto», da qual emerge como subcategoria «apoio formativo». Com menor relevância surgem as categorias «contexto envolvente» e a «sorte».

\section{Discussão}

Este trabalho teve como objetivo identificar carreira e os percursos de árbitros portugueses de futebol que atingiram o nível de excelência e verificar as suas perceções sobre os fatores que lhe permitiram alcançar este patamar.

De um modo geral, apesar da resumida amostra considerada neste estudo, os resultados sugerem que, de um modo geral, os árbitros portugueses que atingem o patamar de excelência trilham percursos distintos ao longo da vida. Numa fase inicial o percurso escolar foi desenvolvido em paralelo com uma prática desportiva e, em alguns casos, com o envolvimento em projetos associativos. O início da carreira de árbitro, assim como o percurso profissional em áreas como a banca, o comércio ou a engenharia, tiveram impacto no percurso escolar estando mesmo na origem do seu fim entre os 18 e os 23 anos, tal como verificado em estudo anteriores com desportistas profissionais (Torregrosa, Ramis, Pallarés, Azócar, \& Selva, 2015) Ocasionalmente os estudos, ao nível superior, foram retomados após o abandono da carreira de árbitro. Verificou-se ainda que a obtenção do estatuto de árbitro internacional provocou constrangimentos no exercício das suas atividades profissionais não relacionada com o desporto, podendo mesmo contribuir para o seu término. Após o término da carreira de árbitros, estes enveredam maioritariamente por prosseguir o seu percurso profissional em atividades relacionadas com a arbitragem.

Os resultados apresentam consistência com os estudos desenvolvidos anteriormente no âmbito do desenvolvimento desportivo e abandono no âmbito da arbitragem. Em comum sugerem um desenvolvimento faseado até um patamar de excelência que antecede o abandono, muitas vezes no desporto (Alfermann \& Stambulova, 2007; Côté \& Vierimaa, 2014; Stambulova, 1994; Stambulova et al., 2009). A dificuldade em conciliar os estudos e uma exigente carreira desportiva está atestada em investigações anteriores (Borggrefe \& Cachay, 2012; Durand-Bush \& Salmela, 2002; Ryan, 2015), principalmente em relação aos jogadores de futebol (Bossle \& Lima, 2013; Carvalho, 2002; Christensen \& Sørensen, 2009)

Relativamente ao segundo objetivo os resultados sugerem que a carreira dos árbitros é influenciada por diversos elementos de índole interna e contextual. Nos primeiros foram percecionadas características individuais de cariz socioemocional. A paixão pelo exercício da atividade de árbitro já tinha sido identificada nos árbitros portugueses (Antonio, Brandão, Serpa, Krebs, \& Araújo, 2011), franceses (Philippe et al., 2009) e noruegueses (Johansen, 2015), assim como a importância do otimismo como estratégia coping para enfrentar com agressões físicas e verbais do meio envolvente (Folkesson et al., 2002). Um estudo qualitativo realizado com árbitros da primeira categoria inglesa já tinha identificado situações específicas que exigiam um esforço mental acrescido, assim como conhecimentos e conhecimentos e comportamentos utilizados para enfrentar estes desafios (Slack, Butt, Maynard, \& Olusoga, 2014). A importância do conhecimento das leis de jogo e de possuir uma boa condição física foi percecionada por árbitros ingleses, sendo que esta última foi identificada nos árbitros espanhóis (Castillo et al., 2016) e ucranianos (Berezka \& Chopilko, 2014). Uma boa 
capacidade motora foi identificada nos árbitros sérvios (Mrkovic, Talavic, Jeleskovic, Alic, \& Bajramovic, 2009). A evolução resultante do treino específico e da direção de jogos, explorado nas abordagens que incidem sobre a expertise e Prática Deliberada,, foi percecionada pelos participantes, tal como tinha ocorrido com os árbitros inglês (Slack et al., 2013). Mais recentemente um estudo qualitativo efetuado junto de 24 especialistas em futebol apontou para a existência de três dimensões distintas na origem da excelência do árbitro: a sua preparação individual, a preparação dos jogos e a gestão dos mesmos (Aragão e Pina, Passos, Carvalho, \& Travis Maynard, 2018).

Como elementos externos considerados relevantes na carreira dos árbitros foram percecionados: (i) a existência uma rede social e de pessoas significativas que ajudem no desenvolvimento da carreira sustentada do árbitro já identificada por árbitros franceses (Perreau-niel \& Erard, 2015) e portugueses (Sarmento et al., 2015) mas também em estudos com futebolistas portugueses (Teques \& Serpa, 2010); (ii) a existência de um apoio institucional, que disponibilize condições adequadas para o desenvolvimento da carreira. Um estudo de caso junto de árbitros turcos demonstrou como este apoio foi decisivo na melhoria da sua capacidade de resistência dos árbitros (Kizilet, 2011); (iii) a vivência de experiências significativas (Gagné, 2000, 2004), como um desempenho desportivo relevante ou a participação em grandes eventos (Stambulova, 1994; Stambulova et al., 2009), à semelhança do que foi identificado em estudos com árbitros da Premier League ingleses (Slack et al., 2014) e de todas as divisões israelitas (Samuel et al., 2015); (iv) a concessão de oportunidades que permitam, por exemplo, a integração em programas específicos de desenvolvimento de talentos (Gagné, 2004; Porath, 2004), como ocorre a nível da UEFA com o programa CORE (Racek \& Pelikán, 2015), ou em ambientes positivos de aprendizagem (Baltes \& Staudinger, 2000; Bloom, 1985; Côté \& Salmela, 1996; Durand-Bush \& Salmela, 2002), como foi apontado em vários trabalhos sobre a arbitragem inglesa (Slack et al., 2013; Webb, 2017; Webb et al., 2016). Paralelamente foram também identificados, como elemento externo que pode dificultar um percurso até a obtenção de um nível de excelência a existência de determinados obstáculos. Um deles decorrente da falta de experiência acumulada para enfrentar transições não normativas como agressões ou descidas de categoria (Samuel et al., 2015). Esta pode ser entendida como elemento externo na medida que a nomeação para jogos é da responsabilidade de estrutura institucional. O outro obstáculo deriva da qualidade limitada e/ou da falta de honestidade dos recursos humanos responsáveis pela avaliação de árbitros identificados pelos árbitros israelitas (Samuel et al., 2015) e pelos árbitros portugueses (Sarmento et al., 2015).

\section{Implicações}

A carreira de árbitro, devido à suas exigências, limita o desenvolvimento de percursos escolares e profissionais paralelos. Neste sentido importa discutir a pertinência da introdução de programas que promovam o desenvolvimento de carreira duplas. Esta pode ser uma solução a proporcionar aos árbitros (Perreau-niel \& Erard, 2015; Racek \& Pelikán, 2015) que não conseguem garantir uma independência eco- nómica através exercício das suas funções ou que, após a sua retirada por volta dos 45 anos, não conseguem dar continuidade a um percurso profissional ligado ao sector. Paralelamente este tipo de programas pode possibilitar um desenvolvimento simultâneo da individualidade do árbitro, tanto a nível pessoal como desportivo, essencial para quem pretende níveis de desempenho de excelência (Carless \& Douglas, 2013).

Noutro sentido os resultados deste estudo vêm reforçar o alerta de alguns autores (Aragão e Pina, Passos, Araújo, et al., 2018; Aragão e Pina, Passos, Carvalho, \& Travis Maynard, 2018) para a pouca atenção atribuída pelos investigadores para a temática organizacional na arbitragem como elemento catalisador ou constrangedor no desenvolvimento do potencial de cada árbitro possibilitando um maior acompanhamento, uma melhor oferta formativa, garantindo uma avaliação justa e um maior número de oportunidades.

Com exceção de um estudo de caso sobre o apoio institucional dado aos árbitros turcos na sua preparação física (Kizilet, 2011), a maioria dos trabalhos existentes apenas se limita a descrever programas de formação de árbitros de futebol (Racek \& Pelikán, 2015) ou a analisar a evolução da rede de apoio para os árbitros de elite desde a sua criação até à introdução da profissionalização da arbitragem (Webb, 2017).

\section{Referências}

Antonio, A., Brandão, R., Serpa, S., Krebs, R., \& Araújo, D. (2011). El significado de arbitrar/ : percepción de jueces de fútbol profesional 1. Revista de Psicología Del Deporte ISSN:, 20(2), 275-286.

Aragão e Pina, J., Passos, A., Araújo, D., \& Maynard, M. T. (2018). Football refereeing: An integrative review. Psychology of Sport and Exercise, Vol. 35, pp. 10-26. https://doi.org/10.1016/j.psychsport.2017.10.006

Aragão e Pina, J., Passos, A. M., Carvalho, H., \& Travis Maynard, M. (2018). To be or not to be an excellent football referee: different experts' viewpoints. Journal of Sports Sciences, 1-9. https://doi.org/10.1080/ 02640414.2018.1522940

Araújo, L., Cruz, J. F., \& Almeida, L. S. (2011). A entrevista no estudo da Excelência: Uma proposta. Psychologica, 52(2003), 253-280.

Baltes, P. B., \& Staudinger, U. M. (2000). Wisdom: A metaheuristic (pragmatic) to orchestrate mind and virtue toward excellence. American Psychologist, 55(1), 122136. https://doi.org/10.1037/0003-066X.55.1.122

Bardin, L. (2004). Análise de Conteúdo. ((3. ${ }^{a}$ Ed.)). Lisboa: Ediç̧̃̃es 70.

Berezka, S. M., \& Chopilko, T. G. (2014). An investigation of individual functionality football referees qualifications. Physical Education of Students, 18(6), 8-12. https:// doi.org/10.15561/20755279.2014.0602

Bloom, B. S. (Ed.). (1985). Developing talent in young people. New York: Ballantine.

Boyko, R. H., Boyko, A. R., \& Boyko, M. G. (2007). Referee bias contributes to home advantage in English Premiership football. Journal of Sports Sciences, 25(11), 1185-1194. https://doi.org/10.1080/02640410601038576 
Carless, D., \& Douglas, K. (2013). «In the boat» but «selling myself short»: stories, narratives, and identity development in elite sport. The Sport Psychologist, 27(1), 27-39.

Castillo, D., Javier, Y., Casajús, J. A., \& Cámara, B. J. (2016). Physical Fitness and Physiological Characteristics of Soccer Referees. Science et Sports, 31(1), 27-35. https:/ /doi.org/10.1016/j.scispo.2015.11.003

Catteeuw, P., Gilis, B., Wagemans, J., \& Helsen, W. F. (2010). Offside decision making of assistant referees in the English Premier League: Impact of physical and perceptual-cognitive factors on match performance. Journal of Sports Sciences, 28(5), 471-481. https:// doi.org/10.1080/02640410903518184

Catteeuw, P., Helsen, W. F., Gilis, B., \& Wagemans, J. (2009). Decision-making skills, role specificity, and deliberate practice in association football refereeing. Journal of Sports Sciences, 27(11), 1125-1136. https://doi.org/ 10.1080/02640410903079179

Constantin, G. (2015). Finding , preparing and training football referees. Sp Soc Int J Ph Ed Sp, 15(1), 44-63.

Côté, J. (1999). The Influence of the Family in the Development of Talent in Sport. The Sport Psychologist, 13, 395-417. https://doi.org/10.1123/tsp.13.4.395

Côté, J., Ericsson, K. A., \& Law, M. P. (2005). Tracing the development of athletes using retrospective interview methods: A proposed interview and validation procedure for reported information. Journal of Applied Sport Psychology, 17, 1-19. https://doi.org/10.1080/ 10413200590907531

Côté, J., Macdonald, D. J., Baker, J., \& Abernethy, B. (2006). When «where» is more important than «when»: Birthplace and birthdate effects on the achievement of sporting expertise. Journal of Sports Sciences, 24(10), 1065-1073. https://doi.org/10.1080/02640410500432490

Côté, J., \& Salmela, J. (1996). The organizational tasks of high-performance gymnastic coaches. The Sport PsychologistSport Psychologist, 10, 247-260. https:// doi.org/10.1123/tsp.10.3.247

Côté, J., \& Vierimaa, M. (2014). The developmental model of sport particiation: 15 years after its first conceptualization. Science \& Sports, 29((Supl.)), 63-69. https://doi.org/ 10.1016/j.scispo.2014.08.133

Dias, C., Cruz, J. F., \& Fonseca, A. M. (2009). Emoções, stress, ansiedade e coping: estudo qualitativo com atletas de elite. Revista Portuguesa de Ciências Do Desporto, 9, 9-23.

Dohmen, T. (2008). The influence of social forces: Evidence from the behavior of football referees. Economic Inquiry, 46(3), 411-424. https://doi.org/10.1111/j.14657295.2007.00112.x

Dohmen, T., \& Sauermann, J. (2016). Referee Bias. Journal of Economic Surveys, 30(4), 679-695. https://doi.org/ 10.1111/joes.12106

Durand-Bush, N., \& Salmela, J. H. (2002). The development and maintenance of expert athletic performance: Perceptions of world and olympic champions. Journal of Applied Sport Psychology, 14, 154-171. https://doi.org/ 10.1080/10413200290103473

Folkesson, P., Nyberg, C., Archer, T., \& Norlander, T. (2002).
Soccer Referees' Experience of Threat and Aggression: Effects of Age, Experience, and Life Orientation on Outcome of Coping Strategy. Aggressive Behavior, 28, 317-327. https://doi.org/10.1002/ab.90028

Furley, P., \& Schweizer, G. (2016). Nonverbal Communication of Confidence in Soccer Referees/ : An Experimental Test of Darwin's Leakage Hypothesis Nonverbal Communication of Confidence in Soccer Referees/ : An Experimental Test of Darwin's Leakage Hypothesis. Journal of Sport \& Exercise Psychology, 38(6), 590597. https://doi.org/10.1123/jsep.2016-0192

Gagné, F. (2000). A Differentiated model of giftedness and talent (DMGT). https://doi.org/10.1177/ 016235329902200209

Gagné, F. (2004). From gifts to talents: The DMGT as a developmental model. High Ability Studies, 15(2), 119147. https://doi.org/10.1017/CBO9780511610455.008

Gallardo, M. A., \& Iglesias, A. J. B. (2011). La integración de tecnología educativa en la formación del arbitraje de fútbol de élite/ : el uso del « Vídeo Test Interactivo .» Apunts. Educació Física y Esports, 105(1), 12-20. https://doi.org/ 10.5672/apunts.2014-0983.es.(2011/3).105.01

González Oya, J. L. (2005). Reflexiones sobre la formación del árbitro: el papel de la psicopedagogía en el sistema educativo arbitral. Revista Galego-Portuguesa de Psicología y Educación, 12(10), 187-194.

González Oya, J. L. (2006). Aproximación a la formación del árbitro de fútbol: un ejemplo de modelo educativo. Cuadernos de Psicología Del Deporte.

Graneheim, U. H., \& Lundman, B. (2004). Qualitative content analysis in nursing research: Concepts, procedures and measures to achieve trustworthiness. Nurse Education Today, 24, 105-112. https://doi.org/10.1016/ j.nedt.2003.10.001

Holt, N. L., \& Dunn, J. G. H. (2004). Toward a grounded theory of the psychosocial competencies and environmental conditions associated with soccer success. Journal of Applied Sport Psychology, 16, 199-219. https://doi.org/ 10.1080/10413200490437949

Jalil, P., \& Boujettif, M. (2005). Some characteristics of Nobel laureates. Creativity Research Journal, 17 (2, 3), 265272.

Johansen, B. T. (2015). Reasons for officiating soccer: the role of passion-based motivations among Norwegian elite and non-elite referees. Movement \& Sport Sciences Science \& Motricité, 30(87), 23-30. https://doi.org/ $10.1051 / \mathrm{sm} / 2014012$

Kizilet, A. (2011). Using distance physical education in elite class soccer referee training: a case study. TOJET: The Turkish Online Journal of Educational Technology, 10(3), 328-339.

Krustrup, P., Helsen, W. F., Randers, M. B., Christensen, J. F., Macdonald, C., Rebelo, A. N., \& Bangsbo, J. (2009). Activity profile and physical demands of football referees and assistant referees in international games. Journal of Sports Sciences, 27(11), 1167-1176. https://doi.org/ 10.1080/02640410903220310

Mrkovic, R., Talavic, M., Jeleskovic, E., Alic, H., \& Bajramovic, I. (2009). Correlation of refereeing motor characteristics and success. Homo Sporticus, 11(2), 19-22. 
Oh, I. S., Wang, G., \& Mount, M. K. (2011). Validity of Observer Ratings of the Five-Factor Model of Personality Traits: A Meta-Analysis. Journal of Applied Psychology, 96(4), 762-773. https://doi.org/10.1037/a0021832

Perreau-niel, A., \& Erard, C. (2015). French football referees/ : an exploratory study of the conditions of access and employment for referees in terms of level and gender. Soccer \& Society, 16(1), 1-16. https://doi.org/10.1080/ 14660970.2012.627168

Philippe, F. L., Vallerand, R. J., Andrianarisoa, J., \& Brunel, P. (2009). Passion in Referees: Examining Their Affective and Cognitive Experiences in Sport Situations. Journal of Sport and Exercise Psychology, 31(1), 77-96. https:// doi.org/10.1123/jsep.31.1.77

Porath, M. (2004). Transforming gifts into talents: The DMGT as a developmental theory - A response. High Ability Studies. https://doi.org/10.1080/1359813042000314718

Racek, O., \& Pelikán, O. (2015). Role of sports management in preparation for football referees in the Czech Republic. Sport Mont Journal, 43(45), 361-368.

Robbins, S. P., \& Judge, T.A. (2013). Organizational behavior (15th ed.). Upper Saddle River, NJ: Pearson/Prentice Hall.

Samuel, R. D., Galily, Y., \& Tenenbaum, G. (2015). Who are you, ref? Defining the soccer referee's career using a change-based perspective. International Journal of Sport and Exercise Psychology, 15(2), 118-130. https:// doi.org/10.1080/1612197X.2015.1079792

Sarmento, H. M., Marques, A., \& Pereira, A. (2015). Representações, estímulos e constrangimentos do árbitro de futebol de 11. Managing Sport and Leisure, 11(4), 15-25. https://doi.org/10.6063/motricidade.3496

Schilling, J. (2006). On the pragmatics of qualitative assessment designing the process for content analysis. European Journal of Psychological Assessment, 22(1), 28-37. https://doi.org/10.1027/1015-5759.22.1.28

Simonton, D. K. (1999). Significant samples: The psychological study of eminent individuals. Psychological Methods, 4 (4), 425-451.

Slack, L. A., Butt, J., Maynard, I., \& Olusoga, P. (2014). Understanding mental toughness in elite football officiating: perceptions of English Premier League referees. Sport and Exercise Psychology Review, 10(1), 4-24.

Slack, L. A., Maynard, I. W., Butt, J., \& Olusoga, P. (2013). Factors Underpinning Football Officiating Excellence:
Perceptions of English Premier League Referees. Journal of Applied Sport Psychology, 25(3), 298-315. https:// doi.org/10.1080/10413200.2012.726935

Stambulova, N. (1994). Developmental sports career investigations in Russia: A post-perestroika analysis. The Sport Psychologist, 8, 221-237. https://doi.org/10.1123/ tsp.8.3.221

Stambulova, N., Alfermann, D., Statler, T., \& Côté, J. (2009). ISSP position stand: Career development and transitions of athletes. International Journal of Sport and Exercise Psychology, 7(4), 395-412. https://doi.org/10.1080/ 1612197X.2009.9671916

Teques, P., \& Serpa, S. (2010). Talentos no futebol/: O papel dos pais. In C. Nogueira, I. Silva, L. Lima, A. T. Almeida, R. Cabecinhas, R. Gomes, ... M. C. Taveira (Eds.), Actas do VII Simpósio Nacional de Investigação em Psicologia (pp. 1962-1976). Retrieved from http:// www.actassnip2010.com

Torregrosa, M., Ramis, Y., Pallarés, S., Azócar, F., \& Selva, C. (2015). Olympic athletes back to retirement: A qualitative longitudinal study. Psychology of Sport and Exercise, 21, 50-56. https://doi.org/10.1016/ j.psychsport.2015.03.003

Webb, T. (2017). Elite refereeing structures in England: a perfect model or a challenging invention? Soccer and Society, 18(1), 47-62. https://doi.org/10.1080/ 14660970.2014.980740

Webb, T., Wagstaff, C. R. D., Rayner, M., \& Thelwell, R. (2016). Leading Elite Association Football Referees: Challenges in the Cross-Cultural Organization of a Geographically Dispersed Group Tom Webb, Christopher R. D. Wagstaff, Mike Rayner \& Richard Thelwell. Managing Sport and Leisure, 21(3), 105-123. https:// doi.org/10.1080/23750472.2016.1209978

Weston, M., Gregson, W., Castagna, C., Breivik, S., Impellizzeri, F. M., \& Lovell, R. J. (2011). Changes in a toplevel soccer referee' s training, match activities and physiology over an 8-year period/ : a case study. International Journal of Sports Physiology and Performance, 6(2), 281-286.

Wicker, P., \& Frick, B. (2016). Recruitment and Retention of Referees in Nonprofit Sport Organizations/ : The TrickleDown Effect of Role. VOLUNTAS: International Journal of Voluntary and Nonprofit Organizations, 27(3), 13041322. https://doi.org/10.1007/s11266-016-9705-4

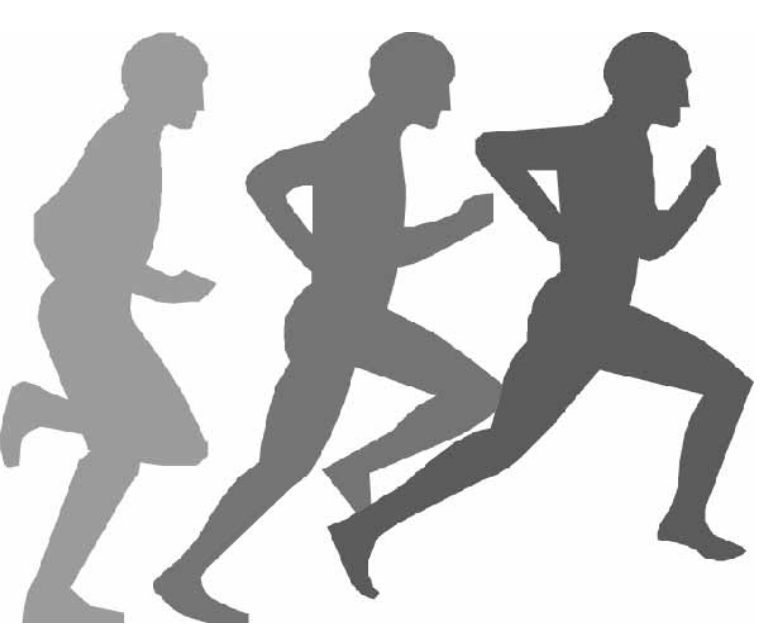

